\title{
CAPRICE D'UNE CANULATION RADIALE
}

\author{
Marcel Boulanger, M.D. ${ }^{*}$
}

(“... où l'on voit que la plus belle fille du monde peut donner parfois plus que ce qu'elle a ...”)

L'utilisation de plus en plus répandue des canulations radiales et leur apparente innocuité, nous amènent à rapporter un incident qui comporte un aspect particulièrement inattendu.

Un malade de 39 ans présente une hémorragie active intra-thoracique à la suite d'un pontage aorto-coronarien. (L'artère mammaire gauche préalablement disséquée s'était avérée inutilisable). On décide de pratiquer une médiastinotomie exploratrice; le malade est porteur d'une canule artérielle placée au cours de la première intervention. Le montage est le suivant : la canule (Argyle Medicut \#20) est reliée au capteur Statham par une ligne artérielle de 6 pieds (Cobe Laboratories Inc.) le raccord canule/ligne artérielle est du type mâle-femelle sans prise "luer-lock".

Le malade est placé dans la position habituelle; c'est-à-dire, décubitus dorsal avec les deux bras en adduction complète le long du corps. On procède d'urgence à l'ouverture du thorax où l'on trouve tout près de 3 litres de sang dans la cavité pleurale. On administre les transfusions selon les lectures de tension artérielle et de tension veineuse; la tension artérielle se lit entre 60 et $80 \mathrm{~mm} \mathrm{Hg}$ avec occasionnellement une lecture à 100-110.

On aperçoit soudain près d'un litre de sang répandu sous les champs opératoires et sur le parquet de la salle d'opération.

La canule artérielle était débranchée mais le jet qui en giclait, conservant son alignement avec la ligne artérielle, laissait voir une lecture pulsatile qui pour être inférieure à la pression réelle n'en avait pas moins toutes les caractéristiques d'une lecture fiable.

\section{Discussion}

Avec ce montage artériel, léventualité d'une disjonction accidentelle ne nous avait pas échappé mais ne nous effrayait pas outre mesure car, croyions-nous, toute disjonction s'accompagnerait immédiatement d'une disparition de la lecture artérielle et le diagnostic serait fait sans retard ...

Cet incident prouve que nous avions tort!

* Département d'anesthésiologie, Institut de Cardiologie de Montréal, 5000 est rue Bélanger, Montréal 410, Qué. 


\section{SUMMary}

We wish to report the case of a patient losing close to one liter of blood through a disconnected radial artery canula. The diagnosis was delayed due to the fact that an arterial pressure was recorded despite the disconnection at the canula/arterial line junction. (The set-up had no "luer-lock" device) : the arterial line was apparently in perfect alignment with the jet of blood squirting from the canula, thus conveying a near normal pressure signal from which the diagnosis of the leak could not be established! 